\title{
Patient and Physician Predictors of Inappropriate Acid-suppressive Therapy (AST) Use in Hospitalized Patients
}

Jagdish S. Nachnani, mo ${ }^{1}$ Deepti Bulchandani, mo² Jill Moormeier, MD, $\mathrm{MPH}^{2}$ John Foxworth, Pharmo ${ }^{2}$

\author{
${ }^{1}$ Department of Gastroenterology and Hepatology, University of Missouri-Kansas City, Kansas City, Missouri. \\ ${ }^{2}$ Department of Internal Medicine, University of Missouri-Kansas City, Kansas City, Missouri.
}

Disclosure: Nothing to report.

BACKGROUND: The use of acid suppressive therapy (AST) in prevention of stress ulcers has been well defined in critical care patients, though its use has become increasingly common in general medicine patients, with little to no supportive evidence. None of the previous studies has examined the patient and physician characteristics of inappropriate AST initiation and use in hospitalized patients. The aim of our study was to identify: (1) the appropriateness of AST in hospitalized patients and the cost associated with inappropriate use; and (2) patient and physician characteristics predicting inappropriate initiation and use of AST.

METHODS: All discharges over a period of 8 consecutive days were selected.

RESULTS: There were 207 patients discharged over a period of 8 days. AST was inappropriately initiated in 92 of 133 (69.2\%) patients included in our study. On univariate analysis, higher hemoglobin value, postgraduate year 1 (PGY-1) residents, physicians with an MD degree, international medical graduates (IMGs), and internal medicine physicians were more likely to prescribe AST inappropriately. On multivariate analysis, a higher hemoglobin value, PGY-1 residents, and MD physicians were factors associated with inappropriate AST use. The total direct patient cost for this inappropriate use was $\$ 8026$, with an estimated annual cost of approximately $\$ 366,000$.

CONCLUSIONS: AST was inappropriately initiated in $69.2 \%$ of patients with increased direct costs of $\$ 8026$. Residents in their first year of training as well physicians with a MD degree are more likely to initiate AST inappropriately. Curtailing the inappropriate use of AST therapy may reduce overall costs for the patient and institution. Journal of Hospital Medicine 2009;4:E10-E14. @ 2009 Society of Hospital Medicine.

KEYWORDS: acid-suppressive therapy, inappropriate use, predictors, prophylaxis, stress ulcers.

The United States spends a larger share of its gross domestic product (GDP) on healthcare than any other major industrialized country. ${ }^{1}$ Expenditures for healthcare represent nearly one-seventh of the nation's GDP, and they continue to be one of the fastest growing components of the federal budget. ${ }^{1}$ Drug expenditures are one of the most rapidly growing components of total healthcare expenditures. ${ }^{2}$ Two of the biggest drivers behind this explosive growth of rising drug expenditures are price and use. ${ }^{2,3}$

Acid-suppressive therapy (AST), including histamine-2 (H2) receptor antagonists and proton pump inhibitors (PPIs), is used extensively in the hospitalized population. ${ }^{4}$ One of the most common uses of AST in hospitalized patients has been in preventing gastric mucosal damage and bleeding. ${ }^{5}$ However, published data suggest that the use of AST will be beneficial only in a well-defined group of critical care patients in preventing stress ulcers and bleeding. ${ }^{6-8}$ This perception of benefit has been extrapolated to hospitalized patients in general, with little or no evidence to support its use. ${ }^{7,9}$

There have only been limited studies on the overall use or the appropriateness of use of AST in hospitalized patients. ${ }^{7,9}$ Also, there have been no studies that have looked at patient or physician factors which can predict the appropriateness of initiation and use of AST in hospitalized patients. The aim of our study was to identify:

1. The appropriateness of acid suppressive therapy in hospitalized patients admitted to a tertiary teaching institution and the associated cost of inappropriate AST use to the patient.

2. Patient and physician characteristics which can predict the inappropriate initiation and use of AST in patients.

\section{Methods}

This study was conducted at a 308-bed tertiary academic medical center. On an average, there are approximately 800 to 1000 discharges every month from this hospital. All consecutive discharges over a period of 8 consecutive days were selected for inclusion in the study. All patients were assessed for the use of AST during their hospitalization. "Use" was defined as any prescription of an acid-suppressive medication, regardless of dosage regimen, in which the patient received at least 1 dose during their hospitalization. ${ }^{7}$ The class of agents prescribed for AST was also noted. Ranitidine is the preferred $\mathrm{H} 2$ receptor antagonist and pantoprazole is the preferred PPI on the hospital formulary. It was also recorded whether the patient was on the medication at the 
time of admission. If the patient was on AST prior to admission, the records of the patient were reviewed for the indication for initiation of the AST. The discharge records of all these patients were also reviewed to determine if the patient was continued on AST even after discharge. Patients who were readmitted during the study period were not recounted.

Since the aim of our study was to evaluate the inappropriate initiation of AST in hospitalized patients, the following patients were excluded from the analysis: patients who were on AST prior to admission; patients who had a valid therapeutic indication for AST; and patients who met valid therapeutic indications for AST, such as intensive care unit (ICU) transfers.

Two physicians reviewed the records in order to determine whether there was any indication for AST use. If there was discordance between the 2 physicians, a third physician reviewed the records to assess the appropriateness of AST. Patient and prescribing physician characteristics were collected to assess the predictors of the use of AST.

We used the guidelines published by the American Society of Health-System Pharmacists (ASHP) to determine appropriateness of gastrointestinal (GI) prophylaxis in patients. ${ }^{10}$

GI prophylaxis was defined as appropriate if: Patient was in the ICU plus 1 of the following ${ }^{10}$ :

1. Coagulopathy (ie, platelet count of $<50,000 \mathrm{~mm}^{3}$ or international normalized ratio of $\geq 1.5$, or an activated partial thromboplastin $\geq 2$ times normal);

2. Mechanical ventilation for $>48$ hours;

3. History of GI ulceration or bleeding within 1 year of admission;

4. Glasgow coma score of $\leq 10$;

5 . Thermal injury to $>35 \%$ of body surface area;

6. Partial hepatectomy;

7. Multiple trauma (injury severity score of $\geq 16$ );

8. Transplantation perioperatively in the ICU;

9. Spinal cord injury;

10. Hepatic failure;

11. Two or more of the following risk factors: sepsis; ICU stay of $>1$ week; occult bleeding lasting at least 6 days; and high-dose corticosteroids ( $>250 \mathrm{mg}$ /day of hydrocortisone or equivalent steroid).

Other indications for the appropriate use of AST were as follows: any documentation of current or past gastroesophageal reflux disease (GERD); active peptic ulcer disease or maintenance therapy in patients with peptic ulcer disease; treatment of esophagitis/gastritis/duodenitis; or patients admitted with upper GI bleeding or melena.

Ranitidine is the preferred $\mathrm{H} 2$ receptor antagonist used at this medical center. The cost to the patient of oral ranitidine was $\$ 8.54$ per day while the cost of intravenous therapy was $\$ 135.00$ per day. Pantoprazole is the preferred PPI used in this hospital. The cost of oral pantoprazole was $\$ 10.57$ per day while the cost of intravenous therapy was $\$ 57.00$ per day (Dr. Joel Reddish, PharmD, Truman Medical Center,
Kansas City, MO; Pharmacy Staff; personal communication, September 25, 2007). The cost of intravenous ranitidine was higher than intravenous pantoprazole since ranitidine had to be infused 3 times per day. The cost of AST was calculated by calculating the total number of days during the admission the person was on AST.

\section{Statistical Analysis}

All results are expressed as means \pm standard deviations (SDs) or actual frequencies. Univariate logistic regression was used to assess for the predictors of inappropriate use of AST. SAS software version 9.1 (SAS Institute, Inc., Cary, NC) was used for statistical analysis. Multiple logistic regression was used for multivariate analysis. All parameters with a $P$ value of $<0.15$ were included in the multiple logistic regression model. Backward elimination was done to identify the best-fitting model for logistic regression.

Previous studies have identified an approximately $50 \%$ excessive use of AST. $6,7,9$

A power analysis was performed based upon an alpha level of 0.05 , use of a 2-sided test, and an expected difference between the 2 groups of $25 \%$ ( $75 \%$ inappropriate use in one group, $50 \%$ inappropriate use in the other). This analysis indicated that 65 patients in each of 2 groups would provide $85 \%$ power to detect differences in the prescribing habits of the providers. Therefore it was decided that all discharges over a period of 8 consecutive days would be included in the analysis to meet the required sample size.

\section{Results}

There were 207 patients in our study cohort. Of the 207 patients, $103(49.8 \%)$ were males and 71 (34.3\%) were Caucasians. Of the 207 patients, AST was used in 164 (79.2\%) of the patients. PPI therapy was used in 126 (60.9\%) of the patients while 38 (18.4\%) of the patients were put on $\mathrm{H} 2$ receptor antagonists. In the study cohort, 51 (24.6\%) of the patients had a current or a past diagnosis of GERD. Of the 207 patients, 35 patients were on a PPI prior to admission and 16 were on a $\mathrm{H} 2$ blocker prior to admission. Table 1 describes the demographic characteristics of the patients.

The most common primary admitting diagnosis was either cardiovascular or gastrointestinal. Table 2 outlines the most common admitting diagnoses of the patients.

To determine the predictors of inappropriate initiation of AST in hospitalized patients, excluding the patients as described in the Methods section, there were 133 patients who met the inclusion criteria for analysis. The reason for inappropriate use of AST in all of the 133 patients included for analysis in our study was for stress ulcer prophylaxis in low-risk patients. AST was inappropriately used in 92 of the 133 patients $(69.2 \%)$. On univariate analysis, physician characteristics predictive for inappropriate AST use were being in an early stage of training, physicians in the medicine specialty and physicians who were international medical graduates (Table 3). As far as patient characteristics were

2009 Society of Hospital Medicine DOI 10.1002/jhm.492 Published online in wiley InterScience (www.interscience.wiley.com). 
TABLE 1. Demographic Characteristics of Study Cohort $(n=207)$

\begin{tabular}{lc} 
& $\begin{array}{c}\text { Means } \pm \text { SD or } \\
\text { Actual Frequencies }\end{array}$ \\
\hline Patient characteristics & \\
1) Age (years) & $49.1 \pm 16.1$ \\
2) Race (C/AA/H/O) & $71 / 118 / 12 / 6$ \\
3) Gender (male/female) & $103 / 104$ \\
4) History of diabetes (\%) & $52(25.1)$ \\
5) History of hypertension (\%) & $116(56.0)$ \\
6) History of CAD (\%) & $34(16.5)$ \\
7) ICU stay (\%) & $15(7.3)$ \\
8) Current or past GERD (\%) & $51(24.6)$ \\
9) Use of PPI/H2 receptor antagonist prior to admission (\%) & $51(24.6)$ \\
10) Clopidogrel use (\%) & $8(3.9)$ \\
11) Aspirin use (\%) & $41(19.8)$ \\
12) Corticosteroid use (\%) & $4(1.9)$ \\
13) Coumadin use (\%) & $8(3.9)$ \\
14) Hemoglobin (gm/dL) & $12.65 \pm 2.55$ \\
15) Platelet count (thousands) & $255 \pm 106$ \\
16) Hospital stay (days) & $4.9 \pm 6.1$ \\
Physician characteristics & \\
1) PGY1 (\%) & $127(61.4)$ \\
2) Medical education (MD) (\%) & $161(77.8)$ \\
3) International Medical Graduates (IMGs) (\%) & $80(38.6)$ \\
4) Specialty (Medicine) (\%) & $158(76.3)$ \\
\hline
\end{tabular}

Abbreviations: AA, African Americans; C, Caucasians; $\mathrm{CAD}$, coronary artery disease; GERD, gastroesophageal reflux disease; $\mathrm{H}$, Hispanics; H2, histamine-2; ICU, intensive care unit; 0 , others or not reported; PGY, postgraduate year; PPI, proton pump inhibitor.

\begin{tabular}{|c|c|}
\hline Diagnoses & $\begin{array}{l}\text { Number of } \\
\text { Patients (\%) }\end{array}$ \\
\hline $\begin{array}{l}\text { 1. Cardiovascular: chest pain/CHF exacerbation/arrhythmias/ } \\
\text { PVD }\end{array}$ & $32(15.5)$ \\
\hline $\begin{array}{l}\text { 2. Gastrointestinal: hematemesis/gastric ulcer/abdominal pain/ } \\
\text { CLD/pancreatitis }\end{array}$ & $32(15.5)$ \\
\hline $\begin{array}{l}\text { 3. Neurologic: syncope/dizziness/stroke/meningitis/altered } \\
\text { mental status/seizures }\end{array}$ & $25(12.0)$ \\
\hline $\begin{array}{l}\text { 4. Pulmonary: asthma/COPD exacerbation/pneumonia/ } \\
\text { empyema }\end{array}$ & $24(11.6)$ \\
\hline 5. Trauma/accidents & $15(7.2)$ \\
\hline 6. Psychiatric: psychoses/suicidal ideation/substance abuse & $14(6.8)$ \\
\hline 7. Infectious: cellulitis/wound infections/ abscesses & $13(6.3)$ \\
\hline 8. Oncology & $12(5.8)$ \\
\hline 9. Hematologic: sickle cell crises/anemia/thrombocytopenia & $10(4.8)$ \\
\hline 10. Renal: renal failure/UTI/hematuria & $8(3.9)$ \\
\hline 11. Surgical & $7(3.4)$ \\
\hline 12. Others & $15(7.2)$ \\
\hline
\end{tabular}

Abbreviations: COPD, chronic obstructive pulmonary disorder; CHF, congestive heart failure; CLD, chronic Liver Disease; PVD, peripheral vascular disease; UTI, urinary tract infection.

concerned, only a higher hemoglobin value was associated with the inappropriate use of AST (see Table 3 for details).

On multivariate analysis, as far as patient characteristics were concerned only a higher hemoglobin value was associ-

\begin{tabular}{|c|c|c|}
\hline Parameter & Hazard Ratios & $95 \%$ CI ( $P$ Value) \\
\hline \multicolumn{3}{|l|}{ Patient characteristics } \\
\hline 1) Age & 1.018 & 0.99-1.04 (0.15) \\
\hline 2) Race & 1.46 & $0.68-3.13(0.32)$ \\
\hline 3) Gender & 1.03 & $0.49-2.16(0.94)$ \\
\hline 4) History of diabetes & 1.62 & $0.63-4.14(0.32)$ \\
\hline 5) History of hypertension & 1.28 & $0.61-2.68(0.52)$ \\
\hline 6) History of CAD & 1.26 & $0.37-4.21(0.71)$ \\
\hline 7) Nursing home resident & 0.44 & $0.03-7.20(0.56)$ \\
\hline 8) Aspirin use & 1.69 & $0.59-4.80(0.33)$ \\
\hline 9) Clopidogrel use & 0.89 & $0.08-10.09(0.92)$ \\
\hline 10) Coumadin use & 1.36 & $0.26-7.04(0.71)$ \\
\hline 11) Hemoglobin & 1.24 & $1.06-1.46(0.006)$ \\
\hline 12) Raised WBC count & 0.81 & $0.32-2.00(0.64)$ \\
\hline 13) Platelets & 1.00 & $0.99-1.001(0.23)$ \\
\hline 14) Length of stay & 1.03 & $0.92-1.15(0.61)$ \\
\hline \multicolumn{3}{|l|}{ Physician characteristics } \\
\hline 15) PGY1 (PGY1 vs. others) & 5.18 & $2.34-11.50(<0.0001)$ \\
\hline 16) Medical education (MD vs. others) & 2.59 & $1.08-6.17(0.03)$ \\
\hline 17) Training (IMG vs.AMG) & 5.34 & $2.05-13.93(0.0006)$ \\
\hline 18) Specialty (medicine vs. others) & 3.81 & $1.70-8.55(0.001)$ \\
\hline
\end{tabular}

Abbreviations: $\mathrm{CAD}$, coronary artery disease; $\mathrm{WBC}$, white blood cell; IMG, international medical graduate; AMG, American medical graduate.

\section{TABLE 4. Multivariate Analysis Associated With the Inappropriate Use of Acid-Suppressive Therapy}

\begin{tabular}{llc} 
Parameter & Hazards Ratio & $\mathbf{9 5 \%}$ CI (P value) \\
\hline 1. Hemoglobin (g/dL) & 1.35 & $1.13-1.62(0.001)$ \\
2. Level of training (PGY-1 vs. others) & 4.98 & $1.94-13.19(0.0008)$ \\
3. Medical education (MD vs. others) & 2.81 & $1.01-7.83(0.048)$ \\
\hline
\end{tabular}

NOTE: Area under the curve $=0.77$.

Abbreviations: $\mathrm{CI}$, confidence interval; PGY, postgraduate year.

ated with inappropriate AST use. Residents who were in their first year of training as well as physicians with a MD degree were more likely to prescribe AST inappropriately (Table 4).

The direct calculated patient cost for AST during this time period was $\$ 8026$. The estimated projected cost for AST over a period of 1 year was $\$ 366,000$.

Out of the 92 patients in whom AST was used inappropriately, $6(6.5 \%)$ of the patients were discharged on an $\mathrm{H} 2$ receptor antagonist while $7(7.6 \%)$ of the patients were discharged on PPI therapy.

\section{Discussion}

Prescription drug expenditures are the most rapidly growing component of health care expenditures. ${ }^{2}$ Two of the biggest drivers behind this explosive growth of rising drug expenditures are price and use. ${ }^{2,3}$ PPIs have constantly figured in 
the national top 20 drug lists for dispensed prescription and drug sales. ${ }^{2}$

This study found a very high frequency of overuse of acid suppressive therapy in hospitalized patients for stress ulcer prophylaxis. Unfortunately, a large majority (69.2\%) of these patients were not at an increased risk of stress-related mucosal ulceration. One of the reasons for this widespread use of AST is the overestimation of the risk of stress-related mucosal ulceration in hospitalized patients. However, the fear of stress-ulcer bleeding seems to largely unjustified, as overall rates of bleeding, as reported previously, have been very low. ${ }^{11}$ Our results are consistent with the few reports on the overuse of AST reported previously. Nardino et al., ${ }^{7}$ in a study of 226 patients, found that $65 \%$ of the patients received AST inappropriately. Also in a study from Italy, Parente et al. ${ }^{9}$ found, in a cohort of 799 hospitalized patients, $68 \%$ of the prescriptions for AST were not appropriate.

To date, there has been limited information available on the prescribing characteristics of the physicians, which may help to clarify the inappropriate use of AST. This study was conducted at a tertiary academic medical center and all the admissions are done by residents. This study is the first study that has tried to examine the physician and patient characteristics behind this phenomenon. In multivariate analysis, we found that residents who were in their first year of residency training were more likely to initiate AST inappropriately. This could be secondary to the fact that most of the residents in their first year of training are given "blanket orders" to put all patients on stress-ulcer prophylaxis. In a study done by Liberman and Whelan ${ }^{12}$ at the University of Chicago Hospitals, it was found that house officers learned about stress-ulcer prophylaxis from their supervising residents. Thus, it is possible that as residents progress through their training, the incidence of inappropriate initiation of stress ulcer prophylaxis decreases. We also found that physicians with an MD degree were more likely to initiate AST inappropriately. The reason behind this not clear, though there may be a difference in the medical education that possibly contributes to this.

One curious finding that was associated with an increased use of AST was a higher hemoglobin level. One possibility is that patients with a low hemoglobin value were more likely to be put on AST appropriately. This could be the reason behind the association of a higher hemoglobin value with inappropriate AST use.

One of the reasons for the widespread use of AST is that most practitioners view AST as harmless. ${ }^{6}$ However, the use of AST is not without risks. Multiple studies in the past have found an increased risk of Clostridium difficile-associated disease in patients on AST. ${ }^{13-16}$ Also, AST has been associated with an increased risk of community-acquired pneumonia ${ }^{17}$ as well as a risk of hip fractures. ${ }^{18}$ These studies demonstrate that the use of AST is not without its risks and there is a potential for increased morbidity as well as indirect costs for the patient and the community as a whole associated with its use.

The direct cost for this inappropriate use of AST over a period of 8 days was $\$ 8026$ in our study, with an estimated annual cost close to $\$ 366,000$. This did not include the cost of patients who were discharged inappropriately with AST. Also, this did not include the indirect costs including the increased risk of community-acquired pneumonias, hip fractures, and Clostridium infections. Thus, it is possible that the costs of inappropriate use of AST may be much higher than reported.

One of the limitations of our study was that this study was conducted at a single teaching hospital; thus, it is possible that the results could be biased by the prescribing habits of a relatively few physicians. However, since we looked at all specialties, we had a large cohort of physicians in our study. Also, previous multicenter studies as well as single center studies have demonstrated similar results in terms of overprescription. ${ }^{7,9,19}$ Also, the economic impact has been calculated by assessing the cost that is billed to the patients. This may be different from the cost of the medicines to the hospital and insurers.

\section{Conclusions}

AST was inappropriately used in $69.2 \%$ of the patients studied, leading to an increased direct patient cost of $\$ 8026$ and projected estimated direct healthcare costs of approximately $\$ 366,000$ over 1 year. Residents in their first year of training and physicians with an MD degree are more likely to initiate AST inappropriately in patients. Curtailing the inappropriate use of AST therapy may reduce overall costs for the patient and institution.

\section{Acknowledgements}

This work was presented in part as an abstract in the Quality Improvement Category at the Missouri State American College of Physicians meeting.

\section{Address for correspondence and reprint requests:}

Jagdish S. Nachnani, MD, University of Missouri-Kansas City, 2301 Holmes Street, Kansas City, MO 64108; Telephone: 816-404-3995; Fax: 814-404-0959; E-mail: jnachnani@yahoo.com Received 21 May 2008; revision received 25 December 2008; accepted 7 January 2009.

\section{References}

1. Agency for Healthcare Research and Quality (AHRQ). Health Care Costs Fact Sheet. Available at: http://www.ahrq.gov/news/costsfact.htm. Accessed March 2009.

2. Fink KS, Byrns PJ. Changing prescribing patterns and increasing prescription expenditures in Medicaid. Ann Fam Med. 2004;2(5):488-493.

3. Dubois RW, Chawla AJ, Neslusan CA, Smith MW, Wade S. Explaining drug spending trends: does perception match reality? Health Aff (Milwood). 2000;19(2):231-239.

4. Strid H, Simren M, Bjornsson ES. Overuse of acid suppressant drugs in patients with chronic renal failure. Nephrol Dial Transplant. 2003;18(3): 570-575.

5. Daley RJ, Rebuck JA, Welage LS, Rogers FB. Prevention of stress ulceration: current trends in critical care. Crit Care Med. 2004;32(10): 2008-2013.

6. Grube RR, May DB. Stress ulcer prophylaxis in hospitalized patients not in intensive care units. Am J Health Syst Pharm. 2007;64(13):1396-1400.

7. Nardino RJ, Vender RJ, Herbert PN. Overuse of acid-suppressive therapy in hospitalized patients. Am J Gastroenterol. 2000;95(11):3118-3122. 
8. Leonard J, Marshall JK, Moayyedi P. Systematic review of the risk of enteric infection in patients taking acid suppression. Am J Gastroenterol. 2007;102(9):2047-2056.

9. Parente F, Cucino C, Gallus S, et al. Hospital use of acid-suppressive medications and its fall-out on prescribing in general practice: a 1month survey. Aliment Pharmacol Ther. 2003;17(12):1503-1506.

10. ASHP Therapeutic Guidelines on Stress Ulcer Prophylaxis. Am J Health Syst Pharm. 1999;56(4):347-379.

11. Qadeer MA, Richter JE, Brotman DJ. Hospital-acquired gastrointestinal bleeding outside the critical care unit: risk factors, role of acid suppression, and endoscopy findings. J Hosp Med. 2006;1(1):13-20.

12. Liberman JD, Whelan CT. Brief report: reducing inappropriate usage of stress ulcer prophylaxis among internal medicine residents. A practicebased educational intervention. J Gen Intern Med. 2006;21(5):498-500.

13. Cunningham R, Dale B, Undy B, Gaunt N. Proton pump inhibitors as a risk factor for Clostridium difficile diarrhoea. J Hosp Infect. 2003;54(3): 243-245.
14. Dial S, Alrasadi K, Manoukian C, Huang A, Menzies D. Risk of Clostridium difficile diarrhea among hospital inpatients prescribed proton pump inhibitors: cohort and case-control studies. CMAJ. 2004;171(1):33-38.

15. Yearsley KA, Gilby LJ, Ramadas AV, Kubiak AV, Fone DL, Allison MC. Proton pump inhibitor therapy is a risk factor for Clostridium difficile-associated diarrhoea. Aliment Pharmacol Ther. 2006;24(4):613-619.

16. Dial S, Delaney JA, Barkun AN, Suissa S. Use of gastric acid-suppressive agents and the risk of community-acquired Clostridium difficile-associated disease. JAMA. 2005;294(23):2989-2995.

17. Laheij RJ, Sturkenboom MC, Hassing RJ, Dieleman J, Stricker BH, Jansen JB. Risk of community-acquired pneumonia and use of gastric acid-suppressive drugs. JAMA. 2004;292(16):1955-1960.

18. Yang YX, Lewis JD, Epstein S, Metz DC. Long-term proton pump inhibitor therapy and risk of hip fracture. JAMA. 2006;296(24):2947-2953.

19. Gullotta R, Ferraris L, Cortelezzi C, et al. Are we correctly using the inhibitors of gastric acid secretion and cytoprotective drugs? Results of a multicentre study. Ital J Gastroenterol Hepatol. 1997;29(4):325-329. 